\title{
RESULTADOS MATERNOS E NEONATAIS EM CENTRO DE PARTO NORMAL INTRA-HOSPITALAR
}

Camila Soares Teixeira ${ }^{1}$, Valdelize Elvas Pinheiro ${ }^{2}$, Iracema da Silva Nogueira ${ }^{2}$

Objetivo: descrever os resultados maternos e neonatais da assistência no Centro de Parto Normal Intra-hospitalar da Maternidade Balbina Mestrinho em Manaus. Método: pesquisa exploratório-descritiva, com abordagem quantitativa, onde realizou-se a análise de 378 (74,85\%) prontuários de partos assistidos entre setembro de 2014 a 2016 . Resultados: A maioria das parturientes (50,79\%) era jovem, entre 18 e 25 anos e multíparas (74,86\%), permaneceram em média 03 horas e 14 minutos em trabalho de parto e acompanhadas (97,35\%). Obteve-se uma taxa de episiotomia de 8,46\% e laceração perineal de 52,37\% predominando as de primeiro grau (38,62\%). A maioria dos recém-nascidos permaneceu em contato pele a pele (86,60\%) e foi realizado o clampeamento tardio do cordão umbilical (77,78\%). A média do Apgar no primeiro minuto fora de 8,72 e no quinto minuto 9,76. Conclusões: Os resultados condizem com o que preconiza o Ministério da Saúde e a Organização Mundial de Saúde. Descritores: enfermagem obstétrica; parto humanizado; políticas públicas de saúde

\section{MATERNAL AND NEONATAL OUTCOMES AT THE IN-HOSPITAL BIRTH CENTER}

Objective: to describe the maternal and neonatal outcomes of the care provided at the In-hospital birth center. Method: an exploratory, descriptive study with quantitative approach where the analysis of 378 (74,85\%) birth assisted medical records was performed from September 2014 to September 2016. Results: the majority of parturients (50,79\%) was young, between 18 and 25 years old and multiparas (74,86\%). The result obtained was an episiotomy rate of 8,46\% and 52,37\% of perineal laceration, predominating the first degree lacerations. The majority of newborns remains in skin-to-kin (86,60\%) and was made the late clamping umbilical cord (77,78\%). The average of Apgar's rate was 8,72 in the first minute and 9,76 in the fifth. Conclusion: The results meet the guidelines adopted by the Ministry of Health and the World Health Organization.

Descriptors: obstetric nursing; humanizing delivery; public health policies

\section{RESULTADOS MATERNOS Y NEONATALES EN CENTRO DE PARTO NORMAL INTRAHOSPITALARIO}

Objetivo: describir los resultados maternos y neonatales de cuidados en el Centro de Parto Normal Intrahospitalario de la Maternidad Balbina Mestrinho en Manaus. Método: estudio exploratorio descriptivo con un enfoque cuantitativo, que llevó a cabo el análisis de 378 (74.85\%) de los nacimientos asistidos, registros a partir de septiembre de 2014 y 2016. Resultados: La mayoría de las madres (50,79\%) eran joven entre 18 y 25 años de edad y multíparas (74,86\%) eran, en promedio, 03 horas y 14 minutos y seguido de trabajo de parto acompañadas (97,35\%). Obtenido como una tasa de episiotomías de $8,46 \%$ y $52,37 \%$ laceración perineal el predominio de primer grado (38,62\%). La mayoría de los recién nacidos se mantuvieron en contacto con la piel (86,60\%) y se llevó a cabo a finales de sujeción del cordón umbilical (77,78\%). El promedio de Apgar en el primer minuto fuera de 8,72 y 9,76 en el quinto minuto. Conclusiones: Los resultados son consistentes con la recomendada por el Ministerio de Salud y la Organización Mundial de la Salud.

Descriptores: enfermeria obstetricia; parto humanizado; las políticas de salud pública

IFundação Oswaldo Cruz, Brasil. E-mail: cst.enfg@gmail.com

${ }^{2}$ Universidade do Estado do Amazonas - UEA. 


\section{INTRODUÇÃO}

O Ministério da Saúde, no âmbito da reformulação da assistência ao parto e da busca pela diminuição das intervenções e cesarianas, promoveu no Sistema Único de Saúde, a criação dos Centros de Parto Normal (CPN)1.2.

Os CPN foram instituídos para garantir a condução fisiológica do trabalho de parto e partos normais sem distócia por enfermeiros obstétricos. Possuem equipe de retaguarda, composta por médicos obstetra, anestesista e pediatra, que atuam apenas se houver necessidade ou solicitação da equipe do CPN. Localizam-se como unidade intra-hospitalar ou nas imediações de uma maternidade de referência ${ }^{1}$.

Buscando evitar as intervenções desnecessárias ao processo fisiológico, faz-se uso das boas práticas de atenção ao parto e nascimento, criadas pela Organização Mundial de Saúde (OMS), com o objetivo de classificar as práticas assistenciais realizadas como úteis, recomendadas ou não recomendas pela OMS. Dentre as práticas recomendadas estão: liberdade de posição e movimento durante o trabalho de parto e parto e os métodos não farmacológicos para alívio da dor. Observou-se que modelos intervencionistas nesse processo fisiológico apresentam resultados maternos $\mathrm{e}$ neonatais adversos e as mulheres mostram-se insatisfeitas com tais procedimentos ${ }^{3}$.

Outra estratégia para humanização do parto foi a criação da Rede Cegonha, que visa garantir à mulher o direito a um planejamento reprodutivo de qualidade, e a atenção humanizada durante a gravidez, parto e puerpério. Buscando a redução da mortalidade, a reestruturação e modernização da rede assistencial à saúde materno-infantil 4 .

Estudar a implementação e atuação dos CPN é essencial para o desenvolvimento da Rede Cegonha em Manaus, bem como para a evolução das técnicas de humanização do préparto, parto e puerpério.

Objetivou-se descrever os resultados maternos e neonatais no Centro de Parto Normal Intra-hospitalar de uma maternidade pública em Manaus.

\section{METODOLOGIA}

Trata-se de estudo descritivo com abordagem quantitativa com coleta de dados retrospectiva, desenvolvido em Manaus, no Centro de Parto Normal Intra-hospitalar (CPNIh) da Maternidade Balbina Mestrinho (MBM), centro de referência na atenção à gestação de alto risco, inaugurada em 1961, e reformada na década de 1990.

Quanto a evolução perante as políticas públicas, em 2002 a Maternidade foi contemplada com o Programa Mãe Canguru, em 2005 tornou-se "Hospital Amigo da Criança", e então, em 2014 inaugurou-se o CPNIh.

O CPNIh é composto por três suítes, equipadas com camas PPP (pré-parto, parto e pós-parto) e berços aquecidos para recepção do recém-nascido e $\mathrm{Ol}$ alojamento conjunto, composto por 03 camas hospitalares. A equipe é composta por 02 enfermeiras obstétricas e 02 técnicos de enfermagem, há colaboração complementar, quando necessário, de um médico pediatra e um médico obstetra. O local também atua como campo de estágio de enfermeiros residentes em enfermagem obstétrica, vinculados aos programas de residência em enfermagem das Universidades Federal e Estadual do Amazonas.

A população do estudo contemplou os prontuários de gestantes atendidas no CPNIh da MBM, totalizando 505 prontuários. A seleção da amostra obedeceu aos seguintes critérios de inclusão: prontuários de gestantes atendidas no CPNIh entre setembro de 2014 até setembro de 2016 e prontuários com registros legiveis e sem rasuras, excluindose prontuários ilegíveis e de mulheres de etnia indígena. Buscou-se constituir-se uma amostra representativa para garantir generalização dos resultados e o entendimento das boas práticas preconizadas. Dessa forma, a amostra constituiu-se de 378 (74,85\%) prontuários.

Ressalta-se que houve perda de prontuários por conta de inadequação de armazenamento e de registro referente ao local de parto.

O instrumento utilizado para a coleta de dados consistiu em um formulário elaborado pelas pesquisadoras, contemplando: dados sociodemográficos, características obstétricas e dados específicos do trabalho de parto, parto e pós-parto.

Os dados foram submetidos a análise estatística descritiva, através da frequência absoluta e relativa das variáveis, classificando-as em suas respectivas categorias e discutidas através dos achados de estudos nacionais e internacionais. Também fora realizado a média aritmética do número de consultas pré-natais, contidas na caderneta da gestante das parturientes.

Por setratar de pesquisa em prontuários de seres humanos, a referida pesquisa atendeu as recomendações da Resolução 466/2012 do Conselho Nacional de Saúde(5). Inicialmente, solicitou-se a autorização da Secretaria de Estado da Saúde, para fins de encaminhamento ao Comitê de Ética em Pesquisa da Universidade do Estado do Amazonas, sendo aprovado mediante o parecer CAAE: 55450716.6.0000.5016. As autoras declaram que não há conflitos de interesse neste estudo.

\section{RESULTADOS}

Observou-se que entre as 378 mulheres atendidas, $50,79 \%$ (192) estavam na faixa etária de 18 a 25 anos, e apenas $5,29 \%$ (20) tinham mais de 35 anos. Em relação a escolaridade, 28,57\% (108) mulheres haviam completado o ensino médio, 
não houve registro de nenhuma parturiente analfabeta. Quanto ao estado civil, houve predominância das solteiras (56,35\%; 213).

Observa-se na tabela 1 as características obstétricas das parturientes, cerca de 24,60\% (93) eram primíparas, sendo o maior percentual (27,51\%; 104) apresentado por aquelas que estavam na segunda gestação, a média de consultas pré-natais foram 6,43 consultas. A idade gestacional mais frequente (61,64\%; 233) foi entre 39 semanas e 39 semanas e 6 dias.

Tabela 1 - Características obstétricas das parturientes. Centro de Parto Normal Intra-hospitalar. Manaus. 2014 2016.

\begin{tabular}{|c|c|c|}
\hline \multicolumn{3}{|l|}{ Variáveis } \\
\hline PARIDADE & Frequência & $\%$ \\
\hline 2 a 4 partos anteriores & 244 & $64,55 \%$ \\
\hline Primipara & 93 & $24,60 \%$ \\
\hline 5 ou mais partos anteriores & 39 & $10,32 \%$ \\
\hline Sem Informação & 2 & $0.53 \%$ \\
\hline TOTAL & 378 & $100,00 \%$ \\
\hline CONSULTAS DE PRÉ-NATAL & Frequência & $\%$ \\
\hline 7 ou mais consultas & 183 & $48,42 \%$ \\
\hline 5 a 6 consultas & 120 & $31,75 \%$ \\
\hline 3 a 4 consultas & 50 & $13,22 \%$ \\
\hline 1 a 2 consultas & 16 & $4,23 \%$ \\
\hline Sem Informação & 9 & $2,38 \%$ \\
\hline TOTAL & 378 & $100,00 \%$ \\
\hline IDADE GESTACIONAL & Frequência & $\%$ \\
\hline $39-40 s 6 d$ & 233 & $61,64 \%$ \\
\hline $37-38 s 6 d$ & 135 & $35,71 \%$ \\
\hline$\leq 4$ sem & 10 & $2,65 \%$ \\
\hline TOTAL & 378 & $100,00 \%$ \\
\hline
\end{tabular}

Sobre os acompanhantes, os companheiros foram maioria em 42,33\% (160) dos atendimentos, apenas 1,06\% (4) parturientes estavam desacompanhadas durante o trabalho de parto. Quanto ao período expulsivo e pós-parto, 97,35\% (368) das pacientes estavam acompanhadas.

A respeito do exame de rotina para detecção de sífilis, a maioria (96,56\%; 365) das parturientes apresentou resultado negativo. Entretanto, 8 pacientes apresentaram resultado positivo, e em 0,79\% (3) prontuários não foi encontrado registro sobre o exame.

Recomenda-se a abertura do partograma no momento de internação da parturiente, 68,78\% (260) destas foram acompanhadas por meio deste instrumento.

Como pode ser visto na tabela 2 , a bola suiça foi o método mais utilizado (58,73\%; 222) como exercício de auxílio para progressão do parto e conforto. Observou-se que 15,87\% (60) das parturientes não utilizou nenhum exercício para progressão do parto.

Tabela 2 - Boas Práticas de relaxamento e conforto das parturientes. Centro de Parto Normal Intra-hospitalar. Manaus. 2014-2016.

\begin{tabular}{|lll|}
\hline Variáveis & Frequência & $\%$ \\
\hline Método & 222 & $58,73 \%$ \\
\hline Bola Suiça & 205 & $54,23 \%$ \\
\hline Cavalinho & 180 & $47,62 \%$ \\
\hline Banho Morno & 172 & $45,50 \%$ \\
\hline Escada de Ling & 68 & $17,99 \%$ \\
\hline Deambulação & 60 & $15,87 \%$ \\
\hline Não utilizou & 23 & $6,08 \%$ \\
\hline Outro & 10 & $2,65 \%$ \\
\hline Massagem & & \\
\hline
\end{tabular}

Quanto a posição durante o período expulsivo, a maioria (58,20\%; 220) adotou a posição semideitada, seguido da posição de cócoras (17,72\%; 67). Na associação entre lacerações e posição adotada, observou-se que a maioria dos partos em posição semideitada (108; 60,67\%) não resultou em lacerações. Enquanto que 37,10\% (82) resultaram em laceração de primeiro grau. Das 19 vezes que a banqueta fora utilizada no período expulsivo, 7 vezes houve laceração de grau 2, correspondendo a $36,84 \%$.

Não houve laceração perineal em 47,09\% (178) das parturientes, como demonstra a tabela 3. A laceração mais frequente foi a de primeiro grau (38,62\%; 146). Sobre a realização da episiotomia, 90,74\% (343) das parturientes não fora exposta a esse procedimento. Na relação entre episiotomia e número de gestas, observou-se maior frequência de episiotomia em primigestas (84,37\%; 27).

Tabela 3 - Condições perineais. Centro de Parto Normal Intra-hospitalar. Manaus. 2014-2016

\begin{tabular}{lll|}
\hline Variáveis & & \\
Episiotomia & Frequência & $\%$ \\
\hline Sem episiotomia & 343 & $90,74 \%$ \\
\hline Médio-lateral & 25 & $6,61 \%$ \\
\hline Mediana & 7 & 1,85 \\
\hline
\end{tabular}




\begin{tabular}{|lll|}
\hline Sem Informação & 3 & $0.79 \%$ \\
\hline Laceração & Frequência & $\%$ \\
\hline Sem lacerações & 178 & $47,09 \%$ \\
\hline Grau 1 & 146 & $38,62 \%$ \\
\hline Grau 2 & 49 & $12,96 \%$ \\
\hline Grau 3 & 3 & $0,79 \%$ \\
\hline Sem Informação & 2 & $0.53 \%$ \\
\hline
\end{tabular}

Não houve intercorrências durante a assistência, em 30,42\% (115) dos partos. Entretanto em 59,79\% (226) dos prontuários, essa informação não estava registrada. Houve 01 caso de hemorragia pós-parto e prolapso de cordão e 03 casos de retenção placentária. As parturientes permaneceram em média 03 horas e 24 minutos em trabalho de parto.

Verificou-se que a maioria dos recém-nascidos (81,22\%; 307) permaneceu em contato pele a pele e fora realizado o corte tardio (58,47\%; 221), porém, não se especificou o tempo de contato e para realização do corte.

Em relação aos RNS, houve leve predominância do sexo feminino 52,12\% (197), e peso médio de 3188,33 Kg. Observouse no primeiro minuto uma média do Índice de Apgar de 8,72 e sobre o quinto minuto de vida, predominou $(91,53 \%$; 346$)$ o índice 10 , sendo a média de 9,76.

Sobre a continuidade assistencial aos recém-nascidos, 96,30\% (364) dos RNs permaneceram em alojamento conjunto, apresentando desfecho favorável, enquanto 3,44\% (13) foram encaminhados para a observação neonatal, por motivos como desconforto respiratório e necessidade do uso de medicações.

Dos 378 partos 98,68\% (373) foram assistidos por Enfermeiras (os) obstétricas (as) e em 1,32\% (05) houve a interação com o profissional médico durante o período expulsivo

\section{DISCUSSÃO}

Os resultados do presente estudo mostram que o perfil sociodemográfico das gestantes assistidas no referido CPNIh é semelhante ao encontrado em outros estudos envolvendo CPN e Casas de Parto, em que a maioria das parturientes são adultas jovens, entre 18 a 25 anos $3,6-8$.

O perfil obstétrico das parturientes vai de encontro com o relatado na literatura, uma vez que diversos estudos apresentam as multiparas como maior percentual assistido nos CPN $(3,6,8)$, já a média de consultas pré-natais assemelhase ao encontrado em estudo paulista, em que $87,3 \%{ }^{9.4,2}$ das parturientes realizaram 6 ou mais consultas 9 . O Ministério da Saúde, recomenda no mínimo 06 consultas de pré-natal, para o alcance de uma assistência de qualidade, com realização de exames e orientações para condução da gestação, parto e puerpério $^{10}$

Apesar da resistência e das dificuldades de implantação da Lei $n^{\circ} 11.108$, a maioria das parturientes estava acompanhada durante o trabalho de parto e partoll.

Sobre os exames para detecção de sífilis, a medida é uma estratégia da Rede Cegonha, para auxiliar a detecção e prevenção da transmissão vertical ${ }^{4,12}$. O Norte é a terceira região brasileira com maior incidência de sífilis congênita ${ }^{12}$, fato preocupante uma vez que relaciona-se com altos índices de internação em Unidade de Terapia Intensiva Neonatal.

A instituição apresenta boa adesão ao partograma, recomendado pela Diretriz Nacional de Assistência ao Parto Normal, ressalta-se que linhas de ação antes de 04 horas de trabalho de parto podem aumentar intervenções obstétricas sem benefícios para o binômio ${ }^{13}$.

A utilização de recursos que auxiliem na promoção do conforto e alívio da dor mostram-se eficientes em CPN e Casas de Parto, compondo a categoria A de boas práticas de atenção ao parto e nascimento, recomendadas pela OMS ${ }^{13-14}$. Observou-se, nesta amostra, predominância da posição semideitada (58,73\%), entretanto, em estudo conduzido no Distrito Federal, a mesma posição fora adotada por apenas $33,5 \%$ das parturientes ${ }^{15}$

Evidências científicas ${ }^{13}$, demonstram que o uso de posição vertical ou lateralizada quando comparadas as posições convencionais (supina ou litotômica) está associada a redução de aproximadamente 4,29 minutos na duração do segundo periodo do trabalho de parto, beneficiando a vitalidade fetal.

Os índices de laceração perineal de primeiro grau e períneo íntegro encontrados neste estudo (85,71\%) foram maiores que os de outros estudos, que encontraram valores acima de $70 \%$ e $66,8 \%(3,9)$. A maior frequência de realização de episiotomia em primigestas (84,37\%; 27), também fora relatado em outros estudos 9.16 .

Diretrizes recentes não recomendam a realização de episiotomia de rotina, pois não há proteção ao assoalho pélvico sendo os riscos de complicações como dispaurenia, edema, deiscência e infecção mais evidentes ${ }^{13}$

O Ministério da Saúde orienta a realização do contato pele a pele após o nascimento, estimulando o aleitamento materno e evitando a separação do binômio na primeira hora, vale ressaltar que tal medida também auxilia na prevenção da hemorragia pós-parto ${ }^{13}$.

A realização do clampeamento tardio do cordão umbilical é recomendado ${ }^{13}$, buscando aumentar os níveis de hemoglobina, através da promoção de maior transfusão placentária, evitando a deficiência de ferro na primeira infância(17-18).

Quanto ao índice de Ápgar, estudos ${ }^{3.9}$ identificaram que entre 99,2\% e 99,9\% dos recém-nascidos apresentaram Apgar 
$>7$ no primeiro minuto e $100 \%$ no quinto minuto. Os índices de transferências neonatais encontrados são levemente superiores aos resultados de outros estudos, que apresentam $1,4 \%$ e $2 \%$ de percentual de transferência em CPN e Casas de Parto 3 .

O atendimento ao parto de baixo risco é previsto pela Lei do exercício da enfermagem, que atribui ao enfermeiro o acompanhamento da evolução e execução do parto sem distócia e dos cuidados puerperais. Assegura-se uma equipe de retaguarda, que prestará pronto atendimento caso o CPN encaminhe ou solicite atendimento 2,19-20

Algumas limitações observadas no presente estudo, dizem respeito ao preenchimento incompleto dos prontuários. O correto registro facilita a realização de pesquisas que posteriormente subsidiarão a adoção de novas políticas de saúde. Outra limitação encontrada, fora a escassez de estudos recentes realizados em CPN no Brasil, e principalmente na região Norte, dificultando a ampla discussão acerca dos resultados encontrados.

\section{CONCLUSÃO}

Os resultados demonstraram que as práticas assistenciais do referido CPNIh são conduzidas de acordo com as diretrizes de assistência ao parto normal e com as políticas de humanização do parto e nascimento. Dessa forma, constantemente busca-se o respeito à dignidade feminina e ao bem-estar do binômio.

Considerando estudos nacionais e alguns internacionais, os resultados maternos e neonatais do CPNIh, não discordam dos resultados apontados, apresentando desfechos favoráveis para as parturientes de baixo risco. Tais resultados devem incentivar a continuidade de pesquisas nos CPN da região norte, sendo de suma importância caracterizar essa assistência para a obtenção de resultados mais conclusivos, pois a melhoria da qualidade da assistência ao binômio mãe e filho está diretamente relacionada às evidências científicas.

\section{REFERÊNCIAS}

1. Brasil. Ministério da Saúde. Portaria $n^{\circ} .11$ de 7 de Janeiro de 2015. Cria o Centro de Parto Normal-CPN, no âmbito do Sistema Único de Saúde. Brasília-DF; 1999.

2. Brasil. Ministério da Saúde. Portaria n.11 de 7 de Janeiro de 2015. Redefine as diretrizes para implantação e habilitação de Centro de Parto Normal (CPN), no âmbito do Sistema Único de Saúde (SUS), para o atendimento à mulher e ao recém-nascido no momento do parto e do nascimento, em conformidade com o Componente Parto e Nascimento da Rede Cegonha, e dispõe sobre os respectivos incentivos financeiros de investimento, custeio e custeio mensal. Brasilia; 2015.

3. Lobo SF, de Oliveira SMJV, Schneck CA, da Silva FMB, Bonadio IC, Riesco MLG. Resultados maternos e neonatais em Centro de Parto Normal peri-hospitalar na cidade de São Paulo, Brasil. Rev Esc Enferm USP 2010;44(3):812-8.

4. Brasil. Ministério da Saúde. Portaria no 1.459 de 24 de Junho de 2011. Institui, no âmbito do Sistema Único de Saúde - SUS - a Rede Cegonha. Brasilia; 2011.

5. Brasil. Ministério da Saúde. Conselho Nacional de Saúde. Resolução no 466 de 12 de dezembro de 2012. Brasilia: 2012

6. Pereira ALDF, Lima TRL De, Schroeter MS, Gouveia MDSF, Nascimento SD Do. Resultados maternos e neonatais da assistência em casa de parto no municipio do Rio de Janeiro. Esc Anna Nery Rev Enferm. [Internet]. 2013 [cited 2017 Abr 10]:17(1):17-23. Avaliable from: http://www.scielo.br/pdf/ean/vl7nl/03.pdf

7. Pereira ALF, Nicácio MC. A escolha pelo atendimento em casa de parto e avaliação do cuidado pré-natal. Revista de Enfermagem da UFSM [Internet]. 2014 [cited 2017 Abr 10]; 4(3): 546-55. Available from: https://periodicos.ufsm.br/index.php/reufsm/ article/view/13268/pdf

8. Schneck CA, Riesco MLG, Bonadio IC, Diniz CSG, De Oliveira SMJV. Resultados maternos e neonatais em centro de parto normal peri-hospitalar e hospital. Rev Saúde Pública. 2012;46(1):77-86.

9. Silva FMB da, Paixão TCR da, Oliveira SMJV de, Leite JS, Riesco MLG, Osava RH. Assistência em um centro de parto segundo as recomendações da Organização Mundial da Saúde. Rev Esc Enferm USP 2013:47(5):1031-8.

10. Brasil. Ministério Da Saúde. Atenção ao pré-natal de baixo risco. Brasilia: Ministério da Saúde, 2012.
11. Brasil. Lei n 11.108, de 7 de abril de 2005. Altera a Lei no 8.080, de 19 de setembro de 1990, para garantir às parturientes o direito à presença de acompanhante durante o trabalho de parto, parto e pós-parto imediato, no âmbito do Sistema Único de Saúde - SUS. Diário Oficial da Uniāo. Brasilia; 2005.

12. Domingues RMSM, Leal M do C. Incidência de sifilis congênita e fatores associados à transmissão vertical da sífilis: dados do estudo Nascer no Brasil. Cad Saude Publica. 2016:32(6):1-12

13. Brasil. Ministério da Saúde. Diretriz Nacional de Assistência ao Parto Normal.Brasilia: Ministério da Saúde: 2016.

14. Mafetoni RR, Shimo AKK. Métodos não farmacológicos para alivio da dor no trabalho de parto: revisão integrativa [Internet]. 2014 [cited 2017 Abr 10]:18(2):505-12 Avaliable from: http://www.reme.org.br/artigo/detalhes/942

15. Schettini NJC, Griboski RA, Faustino AM. Partos normais assistidos por enfermeiras obstétricas: posição materna e a relação com lacerações perineais espontâneas. Rev Enferm UFPE on-line [Internet]. 2017 [cited 2017 Abr 10]; 11 (Supl.2): 932-40. Available from: https://periodicos.ufpe.br/revistas/revistaenfermagem.

16. Riesco MLG, Costa ASC, Almeida SFS, Basile ALO, Oliveira SMJV. Episiotmia, Laceração e Integridade perineal em partos normais: análise de fatores associados. Rev Enferm UERJ [Internet]. 2011 [cited 2017 Abr 10]; 19(1):77-83. Avaliable from: http:// www.facenf.uerj.br/v19nl/v19nlal3.pdf.

17. Vain NE. Em tempo: como e quando deve ser feito o clampeamento do cordão umbilical: será que realmente importa? Rev Paul Pediatr. Associação de Pediatria de São Paulo; 2015:33(3):258-9.

18. Chaparro CM. Timing of umbilical cord clamping: Effect on iron endowment of the newborn and later iron status. Nutr Rev. [Internet]. 2011 [cited 2017 Abr 10]: 69(Suppl. 1):31-36. DOI: $10.1111 / \mathrm{j} .1753-4887.2011 .00430 . x$

19. Brasil. Lei no 7.498, de 25 de junho de 1986. Dispõe sobre a regulamentação do exercicio da Enfermagem e dá outras providências. Brasilia-DF; 1986.

20. Brasil. Ministério da Saúde. Portaria no 4.279, de 30 de dezembro de 2010. Estabelece diretrizes para a organização da Rede de Atenção à Saúde no âmbito do Sistema Único de Saúde (SUS). Diário Oficial da União. Brasilia-DF; 2010. 